\title{
Mass Screening Is Associated with Low Rates of Acute Kidney Injury among COVID-19 Patients in Hong Kong
}

\author{
Kam Wa Chan a Ivan Fan-Ngai Hung ${ }^{\text {b,c }}$ Owen Tak-Yin Tsang d,e Tak Chiu Wu ${ }^{f}$ \\ Eugene Yuk-Keung Tso ${ }^{g}$ Kwok Cheung Lung ${ }^{\text {h }}$ Chung Man Lam ${ }^{d}$ Gary Chi-Wang Chan ${ }^{\mathrm{i}}$

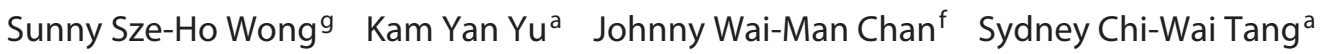 \\ aDivision of Nephrology, Department of Medicine, Queen Mary Hospital, The University of Hong Kong, \\ Hong Kong SAR, China; 'Division of Infectious Diseases, Department of Medicine, Queen Mary Hospital, The \\ University of Hong Kong, Hong Kong SAR, China; 'State Key Laboratory of Emerging Infectious Diseases, Carol Yu \\ Centre for Infection, The University of Hong Kong, Hong Kong SAR, China; ${ }^{d}$ Department of Medicine \& Geriatrics,

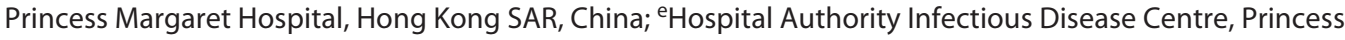 \\ Margaret Hospital, Hong Kong SAR, China; fDepartment of Medicine, Queen Elizabeth Hospital, Hong Kong SAR, \\ China; ${ }^{9}$ Department of Medicine \& Geriatrics, United Christian Hospital, Hong Kong SAR, China; ${ }^{\text {hDepartment of }}$ \\ Medicine, Pamela Youde Nethersole Eastern Hospital, Hong Kong SAR, China; 'Department of Medicine,
}

Queen Mary Hospital, Hong Kong SAR, China

\section{Existing Evidence}

- Most jurisdictions adopted home isolation and admitted only severe COVID-19 patients during the 1st wave. Mass screening and contact isolation are increasingly called for globally. The Hong Kong cohort provided information on the burden of renal complications and associated hospitalization under a vigilant containment policy. Robust contact tracing also allowed characterization of asymptomatic patients and disease course since symptom onset.

\section{Added Value of This Study}

- The Hong Kong cohort was characterized by young age, short time from symptom onset to admission, low acute kidney injury (AKI) rate, low mortality, and long hospitalization.

- The incidence of in-hospital AKI and renal replacement therapy initiation was 3.72 and $0.68 \%$, respectively. AKI increased the odds of prolonged hospitalization and disease course by 2.0 - and 3.5-fold (defined as $>90$ th percentile of hospitalization duration [35 days] and duration from symptom onset to discharge [43 days], respectively).

- Incidence of AKI was comparable between asymptomatic and symptomatic patients.

- There is a trend of persistent glomerular filtration rate (GFR) reduction (defined as post-discharge GFR lower than at admission) after 24 weeks of discharge among patients who developed in-hospital AKI.

- The median time from symptom onset to reaching peak serum $\mathrm{Cr}$ was 15 days among patients who developed inhospital AKI.

- Implications: Among patients who developed in-hospital acute kidney injury (AKI), the duration of hospitalization is prolonged and kidney function impairment can persist for up to 6 months post-discharge. Mass surveillance for COVID-19 is warranted in identifying asymptomatic subjects for earlier AKI management. 


\section{Keywords}

COVID-19 - Renal medicine · Acute kidney injury · Risk

factors $\cdot$ Cohort

\section{Abstract}

Introduction: Renal involvement in COVID-19 is less well characterized in settings with vigilant public health surveillance, including mass screening and early hospitalization. We assessed kidney complications among COVID-19 patients in Hong Kong, including the association with risk factors, length of hospitalization, critical presentation, and mortality. Methods: Linked electronic records of all patients with confirmed COVID-19 from 5 major designated hospitals were extracted. Duplicated records due to interhospital transferal were removed. Primary outcome was the incidence of in-hospital acute kidney injury (AKI). Secondary outcomes were AKI-associated mortality, incident renal replacement therapy (RRT), intensive care admission, prolonged hospitalization and disease course (defined as $>90$ th percentile of hospitalization duration [35 days] and duration from symptom onset to discharge [43 days], respectively), and change of estimated glomerular filtration rate (GFR). Patients were further stratified into being symptomatic or asymptomatic. Results: Patients were characterized by young age (median: 38.4, IQR: 28.4-55.8 years) and short time (median: 5, IQR: 2-9 days) from symptom onset to admission. Among the 591 patients, 22 (3.72\%) developed AKI and $4(0.68 \%)$ required RRT. The median time from symptom onset to in-hospital AKI was 15 days. AKI increased the odds of prolonged hospitalization and disease course by 2.0- and 3.5-folds, respectively. Estimated GFR 24 weeks post-discharge reduced by 7.51 and $1.06 \mathrm{~mL} / \mathrm{min} / 1.73 \mathrm{~m}^{2}$ versus baseline (upon admission) in the AKI and non-AKI groups, respectively. The incidence of AKI was comparable between asymptomatic $(4.8 \%, n=3 / 62)$ and symptomatic $(3.7 \%, n=$ 19/519) patients. Conclusion: The overall rate of AKI among COVID-19 patients in Hong Kong is low, which could be attributable to a vigilant screening program and early hospitalization. Among patients who developed in-hospital AKI, the duration of hospitalization is prolonged and kidney function impairment can persist for up to 6 months postdischarge. Mass surveillance for COVID-19 is warranted in identifying asymptomatic subjects for earlier AKI management.

(c) 2021 S. Karger AG, Basel

\section{Introduction}

Previous reports from Wuhan, China, showed that 75.4\% of COVID-19 patients had abnormal urine dipstick tests or acute kidney injury (AKI), and AKI was associated with 10-fold increase in mortality [1]. However, increasing volume of epidemiological studies suggested that the demographics and clinical presentations varied geographically, partly related to the critical care capacity and the severity of patients being sampled [2-5].

In Hong Kong, the Centre for Health Protection was established after SARS in 2004 to strengthen the surveillance of infectious diseases. Since the outbreak of COVID-19 in December 2019, Hong Kong has maintained a low mortality rate by adopting mass surveillance screening (with compulsory border screening, volunteer community screening, and prehospital screening for all suspected cases), strict border control, and intensive social distancing during the first two waves of pandemics [6]. The contact source of majority of the COVID-19 cases could be traced, tested, identified, and hospitalized for isolation before symptoms develop. Therefore, Hong Kong provided a unique cohort to study the impact of COVID-19 under these public health measures, which are increasingly called for globally to mitigate the transmission [7-9]. The strict contact tracing also allows the characterization of asymptomatic patients.

AKI is common among hospitalized patients, especially for patients under intensive care [10]. Previous studies showed that in-hospital AKI is associated with increased use of renal replacement therapy (RRT) [11] and mortality $[12,13]$. The disease burden of COVID-19 will soon switch from the acute outbreak phase to the end-organ sequela management in the coming months. Therefore, identifying the risk factors and determining the magnitude of AKI incidence in COVID-19 under different degrees of surveillance, public health interventions, and hospital admission policy would help forecasting the expected health services demand in the subsequent waves. We aimed to assess the renal involvement in COVID-19 in Hong Kong, including the association with risk factors, length of hospitalization, critical presentation, and mortality.

\section{Methods}

Study Design

This study is a retrospective cohort analysis of hospital registries with total sampling.
Chan/Hung/Tsang/Wu/Tso/Lung/Lam/ Chan/Wong/Yu/Chan/Tang 


\section{Inclusion and Exclusion Criteria}

All patients aged 18 or above with confirmed COVID-19 (by nasopharyngeal swab, throat swab, or posterior oropharyngeal saliva with RT-PCR testing), both symptomatic and asymptomatic, regardless of screening reason from 15 January to 31 May 2020 admitted to 5 major designated hospitals receiving COVID-19 patients in Hong Kong (Queen Mary Hospital, Princess Margaret Hospital, Queen Elizabeth Hospital, United Christian Hospital, and Pamela Youde Nethersole Eastern Hospital) were included.

\section{Outcome Measures}

The primary outcome was AKI during hospitalization (defined according to KDIGO 2012 guideline as $\geq 26.5 \mu \mathrm{mol} / \mathrm{L}$ [within $48 \mathrm{~h}$ ] or $50 \%$ [within 7 days] increase in baseline serum $\mathrm{Cr}$ [SCr], or urine volume $<0.5 \mathrm{~mL} / \mathrm{kg} / \mathrm{h}$ for $6 \mathrm{~h}$ ) [14]. Secondary outcomes included (1) mortality, (2) incident RRT (including any form of dialysis), (3) critical presentation (defined as intensive care admission) during hospitalization, (4) prolonged hospitalization ( $\geq 90$ th percentile of duration from admission to discharge, 35 days), (5) prolonged disease course ( $\geq 90$ th percentile of duration from symptom onset to discharge, 43 days), and (6) the change of $\mathrm{SCr}$ and estimated glomerular filtration rate (GFR) 4, 12, and 24 weeks after discharge. As the disease course and hospitalization period varied among difference clinical settings, depending on the criteria of screening and discharge with no universal definition, we defined the prolonged strata according to the 90th percentile of the sample population to better stratify according to the clinical need. Estimated GFR was calculated by CKD-EPI equation [15].

Earliest tests taken within $24 \mathrm{~h}$ of admission were defined as baseline, as majority of our patients did not have prehospital SCr record. Discharge investigation was defined as the last test performed before discharge. Disease course was defined as the time from symptom onset to discharge. Patients were discharged after repeated negative RT-PCR.

\section{Data Collection}

Data were extracted through an electronic clinical management system with data linkage to all clinical, laboratory, imaging, and pharmacy records. Smoking history was ascertained by nursing record on admission. All data extraction was entered with a standardized data entry form, and verified by two researchers independently (K.Y.Y., P.Y., and K.W.C.) with at least one physician and clinical epidemiologist (K.W.C.). Duplicated records due to hospital transferal were identified and integrated by unique personal identifier. Outcomes were identified through consultation notes, laboratory investigations, and pharmacy record screening, and the records were last updated on 19 October 2020.

\section{Data Analysis}

Demographics were presented as mean \pm standard deviation, median [interquartile range], or percentage. Differences in median, mean, and proportion between groups, including the difference in demographics and renal manifestations between (1) symptomatic versus asymptomatic patients and (2) patients admitted during the 1 st versus 2 nd waves of COVID-19, were tested by Wilcoxon rank sum test, $t$ test, and $\chi^{2}$ test.

AKI was further stratified into stage 1 ( $\mathrm{SCr} \geq 1.5$ times baseline or increase of $\geq 0.3 \mathrm{mg} / \mathrm{dL}$ within any 48 -h period or urine volume $<0.5 \mathrm{~mL} / \mathrm{kg}$ for $6-12 \mathrm{~h}$ ), stage 2 ( $\mathrm{SCr} \geq 2.0$ times baseline or urine volume $<0.5 \mathrm{~mL} / \mathrm{kg}$ for $\geq 12 \mathrm{~h}$ ), and stage 3 ( $\mathrm{SCr} \geq 3.0$ times baseline or increase to $\geq 4.0 \mathrm{mg} / \mathrm{dL}$ or acute dialysis, or urine volume $<0.3$ $\mathrm{mL} / \mathrm{kg}$ for $\geq 24 \mathrm{~h}$ ) [14]. AKI stages 2 and 3 were combined as secondary analysis when the strata were too small for odds ratio calculation. Diabetes history was stratified into nondiabetic (normal blood glucose at discharge without use of any antidiabetic agents) and diabetic (with the use of antidiabetic agent at baseline). Diabetes history was mainly determined by pharmacy record, as glycated hemoglobin was not routinely tested for COVID-19. Fasting blood glucose measures during hospitalization may represent transient change and are not an ideal diagnosis for diabetes. Hypertension history was stratified into non-hypertensive (without use of any antihypertensive agents) and hypertensive (with the use of hypertensive agent at baseline). Smoking history was stratified into nonsmoker, ex-smoker, and current smoker. Patients admitted on or before 22 February 2020 were classified as cases in the 1 st wave and who admitted after 22 February 2020 were cases in the 2nd wave [16].

Univariable regression models were used to assess the odds ratios of different independent risk factors (demographics: age, gender, history and duration of smoking, and alcohol consumption; physical measurement: blood pressures and oxygen saturation; biochemical and hematological investigations: SCr, estimated GFR, C-reactive protein, creatine kinase, serum albumin, lactate dehydrogenase [LDH], alanine aminotransferase, alkaline phosphatase, hemoglobin, leukocyte, neutrophil, lymphocyte, and platelet; and intervention: use of ACE inhibitor/ARB, antivirals, antibiotics, and glucocorticoids) associated with dependent variable (AKI) individually. Multivariable regression models adjusting age and baseline GFR were used to assess the risk factors individually.

We also assessed the association between all AKI and mortality, critical presentation, length of hospitalization, and length disease course. The changes in SCr and estimated GFR from baseline to discharge, and from baseline to 4,12, and 24 weeks after discharge were compared between the AKI and non-AKI groups by paired $t$ test. Change score approach by paired $t$ test was used instead of mixed model, and missing data were not imputed to provide a more illustrative presentation of the actual change in GFR and prevent over-fitting of data. All analyses were computed using STATA 15.1 .

\section{Results}

\section{Demographics}

The flow of data extraction is summarized in Figure 1. 644 records were identified from total sampling of 5 (out of 7) major designated hospitals receiving COVID-19 cases. Seven duplicated (interhospital transferal), 10 nonretrievable (transit patients), and 36 pediatric patient records were removed. 591 consecutive adult patient records were available for analysis, representing 54.5\% $(591 / 1,085)$ of all Hong Kong cases by 31 May 2020 [16].

The median hospitalization duration was 18 [12-26] days (Table 1). The median age was 38.4 [28.4-55.8] years. In all, $5.9,13.5$, and $9.3 \%$ of patients had a his- 


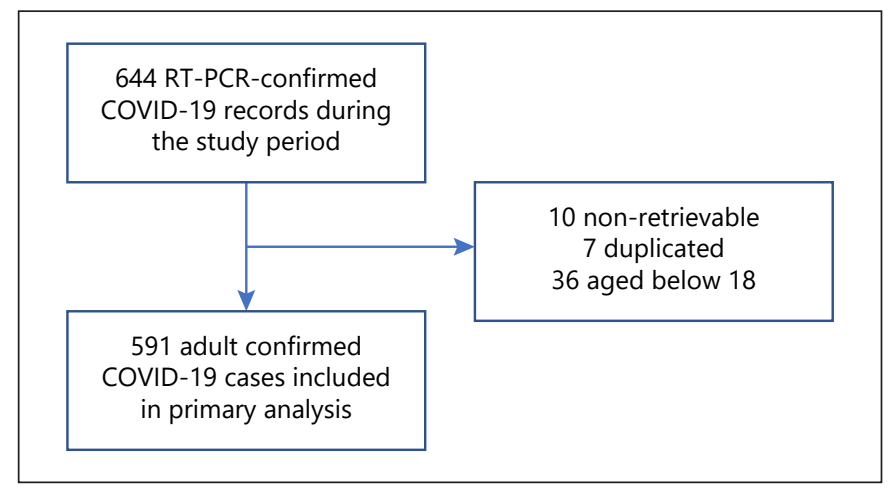

Fig. 1. Flow diagram of data extraction. A total sampling of 644 confirmed COVID-19 records were identified from 5 hospitals. Seven duplicated records of interhospital transferal, 10 non-retrievable records of transit patients, and 36 pediatric patient records were removed. 591 consecutive adult patient records were available from the electronic medical system, representing $54.5 \%$ $(591 / 1,085)$ of all the Hong Kong cases by 31 May 2020 from the 5 (out of 7) major hospitals receiving COVID-19 cases.

tory of diabetes, hypertension, and CKD, respectively; $32.7 \%(166 / 507)$ patients were shown to have bilateral lung involvement by chest X-ray or computed tomography. The median time from symptom onset to admission and discharge was 5 [2-9] and 26 [19-34] days; $10.7 \%$ of patients were asymptomatic, and $20.8 \%$ of patients were diagnosed based on RT-PCR tests before onset of symptoms. The most frequent symptoms were cough $(60.5 \%)$, fever $(60.2 \%)$, fatigue $(46.4 \%)$, sore throat $(39.8 \%)$, and myalgia $(38.4 \%)$. The median SCr and estimated GFR on admission were 72 [61-86] $\mu \mathrm{mol} / \mathrm{L}$ and $101.8[88.6-115.1] \mathrm{mL} / \mathrm{min} / 1.73 \mathrm{~m}^{2}$, respectively. The median peak SCr were 157 [106-254] and 80 [68-95] $\mu \mathrm{mol} / \mathrm{L}$ among the AKI and non-AKI patients, respectively $(p<0.001)$.

\section{Renal and Other Key Clinical Outcomes}

In all, 3.7 and $0.7 \%$ of the patients had AKI and RRT during hospitalization (Table 2); $4.6 \%$ of all patients were admitted to intensive care unit. The median time from symptom onset and admission to reaching peak SCr was 15 [10-18] and 5 [5-12] days, respectively, among patients who developed AKI. AKI developed 2 [1-7] days after admission. Thirteen, 3 , and 6 patients had AKI of stages 1,2 , and 3 , respectively.

Compared to non-AKI patient group, patients who developed in-hospital AKI were older; had higher baseline systolic blood pressure; had higher levels of C-reactive protein, LDH, and creatine kinase; had lower levels of estimated GFR and serum albumin; and had diabetes, hypertension, CKD, cerebrovascular disease, and tumor history. The median time from symptom onset and admission to reaching peak LDH (285 [207-685] U/L) was 9 [8-15.5] and 4 [2-11] days among patients who developed AKI, respectively.

Four patients deceased. The 90th percentiles of disease course and hospital stay were 43 and 35 days, respectively. Patients who developed AKI were associated with 2 times $(\mathrm{OR}=2.99,95 \% \mathrm{CI}: 1.19-7.49, p=0.02)$ and 3.5 times $(\mathrm{OR}=4.48,95 \% \mathrm{CI}: 1.64-12.29, p=0.004)$ increased odds of prolonged hospitalization and disease course (Table 3), respectively.

\section{Longitudinal Renal Function after Discharge}

The mean absolute difference in GFR between admission and discharge was -9.11 and $1.18 \mathrm{~mL} / \mathrm{min} / 1.73 \mathrm{~m}^{2}$ among the AKI and non-AKI groups $(p=0.064)$, respectively (Fig. 2). 242 patients attended 12 -week post-discharge follow-up (median: 77.5 days, IQR: [70-86]). The mean absolute difference in GFR from admission was -3.03 and $-2.41 \mathrm{~mL} / \mathrm{min} / 1.73 \mathrm{~m}^{2}$ among the AKI and non-AKI groups $(p=0.919)$, respectively. 100 patients attended 24-week post-discharge follow-up (median: 163 days, IQR: [146-181]). The mean absolute difference in GFR from admission was -7.51 and $-1.06 \mathrm{~mL} / \mathrm{min} / 1.73$ $\mathrm{m}^{2}$ among the AKI and non-AKI groups $(p=0.301)$, respectively.

\section{Risk Factors for Developing AKI in the Hong Kong Cohort}

Univariable regression analysis showed that older age; smoking; diabetes; hypertension, CKD; tumor history; use of ACE inhibitor/ARB; high baseline systolic blood pressure, C-reactive protein, $\mathrm{LDH}$, and creatine kinase; low baseline serum albumin and estimated GFR; and bilateral lung infiltration were associated with in-hospital AKI in the Hong Kong cohort (Table 4). Patients with Creactive protein $>20 \mathrm{mg} / \mathrm{L}$ or $\mathrm{LDH}>280 \mathrm{U} / \mathrm{L}$ during hospital stay were associated with 2.49 times $(\mathrm{OR}=3.492$, 95\% CI: $1.477-8.255, p=0.004)$ and 4.18 times $(\mathrm{OR}=$ $5.182,95 \%$ CI: $2.141-12.544, p<0.001)$ increased odds of developing AKI. After adjusting for age and baseline GFR, smoking, diabetes, hypertension, use of ACE inhibitor/ARB, high baseline C-reactive protein, $\mathrm{LDH}$, and creatine kinase, low baseline serum albumin, and bilateral lung infiltration remained as significant independent risk factors (online suppl. S2; for all online suppl. material, see www.karger.com/doi/10.1159/000514234). 
Table 1. Characteristics of the Hong Kong cohort

\begin{tabular}{|c|c|c|c|c|}
\hline Characteristic & $\begin{array}{l}\text { All cases } \\
(n=591)\end{array}$ & $\begin{array}{l}\text { AKI during } \\
\text { hospitalization } \\
(n=22)\end{array}$ & $\begin{array}{l}\text { No AKI during } \\
\text { hospitalization } \\
(n=569)\end{array}$ & $\begin{array}{l}p \text { value } \\
\text { (AKI vs. } \\
\text { non-AKI) }\end{array}$ \\
\hline Age, median [IQR], years & $38.4[28.4-55.8]$ & $61.4[42.4-69.3]$ & $38.1[27.9-54.6]$ & $<0.001$ \\
\hline Female, $n(\%)$ & $275(46.5)$ & $9(40.9)$ & $266(46.8)$ & 0.590 \\
\hline Current smoking, $n(\%)$ & $38(6.4)$ & $0(0)$ & $38(6.7)$ & 0.007 \\
\hline \multicolumn{5}{|l|}{ Known comorbidities, $n(\%)$} \\
\hline Diabetes & $35(5.9)$ & $7(31.8)$ & $28(4.9)$ & $<0.001$ \\
\hline Hypertension & $80(13.5)$ & $12(54.6)$ & $68(12.0)$ & $<0.001$ \\
\hline Chronic lung disease & $39(6.6)$ & $2(9.1)$ & $37(6.5)$ & 0.631 \\
\hline CKD & $55(9.3)$ & $6(27.3)$ & $49(8.6)$ & 0.003 \\
\hline Coronary artery disease & $14(2.4)$ & $1(4.6)$ & $13(2.3)$ & 0.494 \\
\hline Cerebrovascular disease & $1(0.2)$ & $1(4.6)$ & $0(0)$ & $<0.001$ \\
\hline Tumor & $14(2.4)$ & $2(9.1)$ & $12(2.1)$ & 0.035 \\
\hline \multicolumn{5}{|l|}{ Days from symptom onset to } \\
\hline admission, median $[\mathrm{IQR}]$ & $5[2-9]$ & $7[4-9]$ & $5[2-9]$ & 0.325 \\
\hline \multicolumn{5}{|c|}{ Signs and symptoms on admission, $n / N(\%)$} \\
\hline Fever & $313 / 520(60.2)$ & $11(50)$ & $302(60.6)$ & 0.318 \\
\hline Cough & $291 / 481(60.5)$ & $16(80)$ & $275(59.7)$ & 0.068 \\
\hline Fatigue/malaise & $83 / 179(46.4)$ & $6(60)$ & $77(45.6)$ & 0.374 \\
\hline Shortness of breath & $56 / 291(19.2)$ & $5(33.3)$ & $51(18.5)$ & 0.155 \\
\hline Chills & $60 / 178(33.7)$ & $3(50)$ & $57(33.1)$ & 0.390 \\
\hline Sore throat & $130 / 327(39.8)$ & $4(33.3)$ & $126(40)$ & 0.643 \\
\hline Sputum & $127 / 337(37.7)$ & $4(26.7)$ & $123(38.2)$ & 0.368 \\
\hline Myalgia & $79 / 206(38.4)$ & $3(37.5)$ & $76(38.4)$ & 0.960 \\
\hline Nausea & $14 / 172(8.1)$ & $1(16.7)$ & $13(7.8)$ & 0.437 \\
\hline Vomiting & $15 / 273(5.5)$ & $0(0)$ & $15(5.7)$ & 0.437 \\
\hline Diarrhea & $117 / 399(29.3)$ & $6(40)$ & $111(28.9)$ & 0.355 \\
\hline Rhinorrhea & $101 / 285(35.4)$ & $3(30)$ & $98(35.6)$ & 0.714 \\
\hline Asymptomatic & $62 / 581(10.7)$ & $3(13.6)$ & $59(10.6)$ & 0.646 \\
\hline \multicolumn{5}{|l|}{ Vital signs on admission, mean (SD) } \\
\hline Systolic blood pressure, $\mathrm{mm} \mathrm{Hg}$ & $137.9(19.2)$ & $148.4(20.9)$ & $137.4(19.0)$ & 0.031 \\
\hline Diastolic blood pressure, $\mathrm{mm} \mathrm{Hg}$ & $84.8(12.0)$ & $87.9(9.5)$ & $84.7(12.1)$ & 0.155 \\
\hline Heart rate, bpm & $85.8(15.0)$ & $87.6(16.6)$ & $85.715 .0)$ & 0.606 \\
\hline Respiratory rate, breaths/min & $16.5(7.8)$ & $16.9(4.3)$ & $16.5(8.0)$ & 0.783 \\
\hline Oxygen saturation, $n(\%)$ & $98.1(2.0)$ & $97.7(1.6)$ & $98.1(2.0)$ & 0.247 \\
\hline \multicolumn{5}{|c|}{ Laboratory findings on admission, 1 median [IQR] } \\
\hline Hemoglobin, g/dL & $13.9[12.9-15]$ & $13.3[12.4-14.4]$ & $13.9[12.9-15]$ & 0.118 \\
\hline Leukocyte, $\times 10^{9} / \mathrm{L}$ & $5.4[4.3-6.8]$ & $4.8[4.1-7.2]$ & $5.4[4.3-6.8]$ & 0.597 \\
\hline Neutrophils, $\times 10^{9} / \mathrm{L}$ & $3.3[2.4-4.5]$ & $3.4[2.4-5.2]$ & $3.3[2.4-4.5]$ & 0.911 \\
\hline Lymphocytes, $\times 10^{9} / \mathrm{L}$ & $1.4[1-1.9]$ & $1.1[0.9-1.6]$ & $1.4[1.0-1.9]$ & 0.076 \\
\hline Platelets, $\times 10^{9} / \mathrm{L}$ & $219[178-271]$ & $187[158-236]$ & $221[179-271]$ & 0.063 \\
\hline Alanine aminotransferase, U/L & $23[16-34]$ & $33.5[17-42]$ & $23[16-34]$ & 0.110 \\
\hline Alkaline phosphatase, U/L & $60[50-74]$ & $67.5[51-76]$ & $60[50-74]$ & 0.468 \\
\hline C-reactive protein, $\mathrm{mg} / \mathrm{L}$ & $2[0-9]$ & $10.8[1.3-56.2]$ & $2[0-8.2]$ & 0.002 \\
\hline Lactate dehydrogenase, U/L & $179[155-217]$ & $241[177-306]$ & $178[155-215]$ & $<0.001$ \\
\hline Bilirubin, $\mu \mathrm{mol} / \mathrm{L}$ & $7[5-10]$ & $8[6-10]$ & $7[5-10]$ & 0.117 \\
\hline $\mathrm{Cr}, \mu \mathrm{mol} / \mathrm{L}$ & $72[61-86]$ & $77.5[57-92]$ & $72[61-86]$ & 0.494 \\
\hline GFR (CKD-EPI), mL/min/1.73 $\mathrm{m}^{2}$ & $101.8[88.6-115.1]$ & $86.4[72.9-104.5]$ & $101.8[89.1-115.7]$ & 0.008 \\
\hline Serum albumin, $g / L$ & $42[38-45]$ & $37.5[35-42]$ & $42[38-45]$ & 0.001 \\
\hline Creatine kinase, $\mathrm{U} / \mathrm{L}$ & $82[58-122]$ & $122.5[58-324]$ & $82[58-120]$ & 0.037 \\
\hline \multicolumn{5}{|l|}{ Radiological findings, $n / N(\%)$} \\
\hline Bilateral infiltrate & $166 / 507(32.7)$ & $14(73.7)$ & $152(31.2)$ & $<0.001$ \\
\hline Right lobes infiltrate & $230 / 507(45.4)$ & $16(84.2)$ & $214(43.9)$ & 0.001 \\
\hline Left lobes infiltrate & $196 / 507(38.7)$ & $15(78.9)$ & $181(37.1)$ & $<0.001$ \\
\hline Any abnormalities & $260 / 507(51.3)$ & $17(89.5)$ & $243(49.8)$ & 0.001 \\
\hline
\end{tabular}


Table 1 (continued)

\begin{tabular}{lcccc}
\hline Characteristic & $\begin{array}{l}\text { All cases } \\
(n=591)\end{array}$ & $\begin{array}{l}\text { AKI during } \\
\text { hospitalization } \\
(n=22)\end{array}$ & $\begin{array}{l}\text { No AKI during } \\
\text { hospitalization } \\
(n=569)\end{array}$ & $\begin{array}{l}p \text { value } \\
\text { (AKI vs. } \\
\text { non-AKI) }\end{array}$ \\
\hline Concurrent treatment, $n / N(\%)$ & $41 / 591(6.9)$ & $6(27.3)$ & $35(6.2)$ & $<0.001$ \\
ACE inhibitor/ARB & $399 / 591(67.5)$ & $22(100)$ & $377(66.3)$ & 0.001 \\
Antivirals & $365 / 591(61.8)$ & $22(100)$ & $343(60.3)$ & $<0.001$ \\
$\quad$ Lopinavir/ritonavir & $328 / 591(55.5)$ & $17(77.3)$ & $311(54.7)$ & 0.036 \\
$\quad$ Ribavirin & $195 / 591(33.0)$ & $8(36.4)$ & $187(32.9)$ & 0.732 \\
$\quad$ Interferon & $170 / 591(28.8)$ & $10(45.5)$ & $160(28.1)$ & 0.078 \\
Antibiotics & $47 / 591(8.0)$ & $1(4.6)$ & $46(8.1)$ & 0.547 \\
Glucocorticoids & & & & \\
\hline
\end{tabular}

AKI, acute kidney injury; GFR, glomerular filtration rate. ${ }^{1}$ Within $48 \mathrm{~h}$ of admission. Among patients with AKI, 13, 3, and 6 had AKI stages 1, 2, and 3, respectively.

Table 2. Clinical outcomes and association between peak serum $\mathrm{Cr}$ and key biomarkers

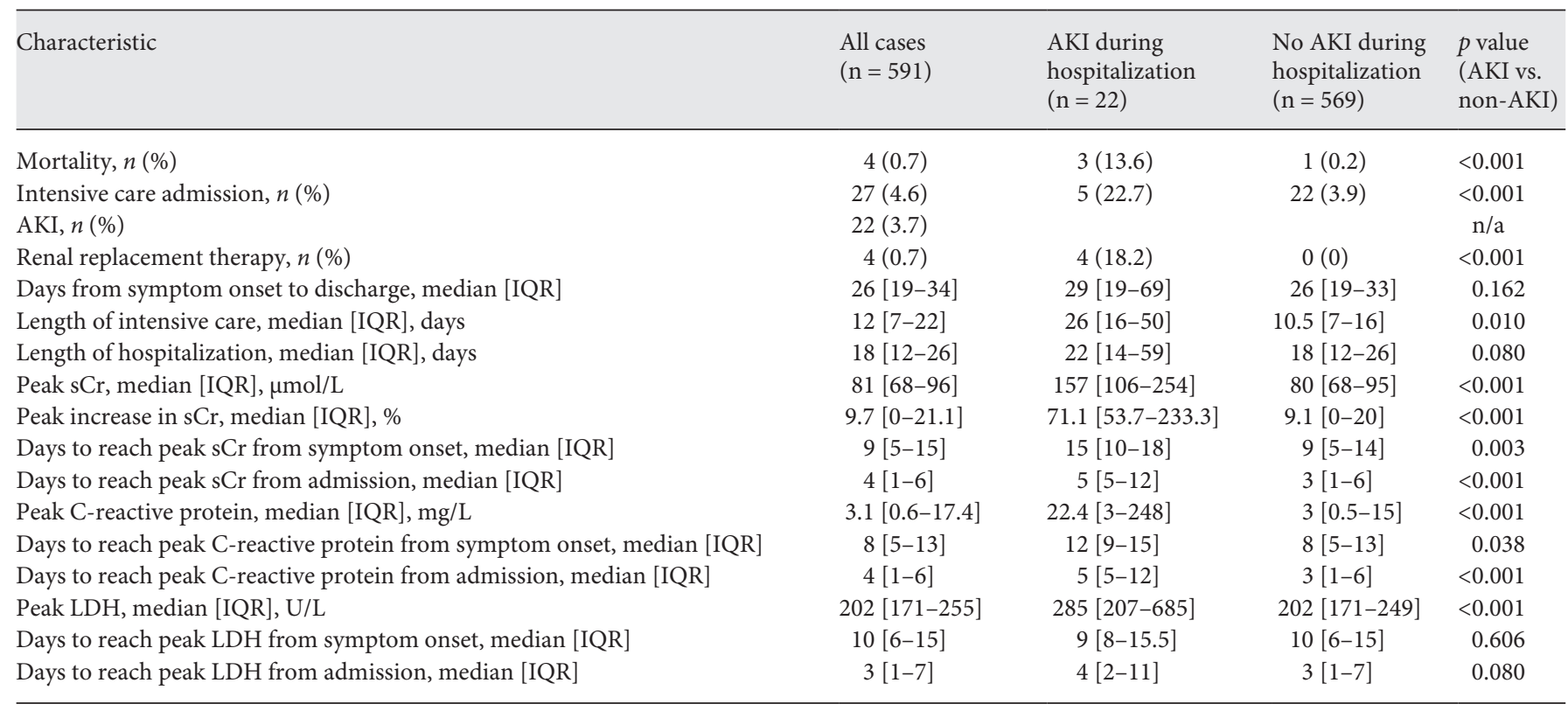

Among patients with AKI, 13, 3, and 6 had AKI stages 1, 2, and 3, respectively. AKI, acute kidney injury; sCr, serum Cr; LDH, lactate dehydrogenase.

\section{Differences in Renal Manifestations between}

Asymptomatic and Symptomatic Patients

Sixty-two patients were asymptomatic before admission. Asymptomatic patients were characterized by less bilateral lung infiltration (asymptomatic: $11.5 \%$ vs. symptomatic: $35.2 \%, p=0.001$ ) and lower C-reactive protein level (asymptomatic: 0.3 vs. symptomatic: $2.3 \mathrm{mg} / \mathrm{L}, p<$ 0.001) (online suppl. S3, S4). The incidence of AKI was comparable between asymptomatic (4.8\%) and symp- tomatic (3.7\%) patients. Asymptomatic patients had a shorter hospitalization period (13 vs. 19 days, $p<0.001$ ) (Table 5).

\section{Differences in Renal Manifestations between Two Waves}

The 2 nd wave $(n=539)$ was characterized by a younger average age ( 1 st: 59.0 vs. 2 nd: 37.4 years, $p<0.001$ ) and with less comorbidities (online suppl. S5, S6). There is a 
Table 3. Prognosis associated with different stages of AKI (Hong Kong cohort)

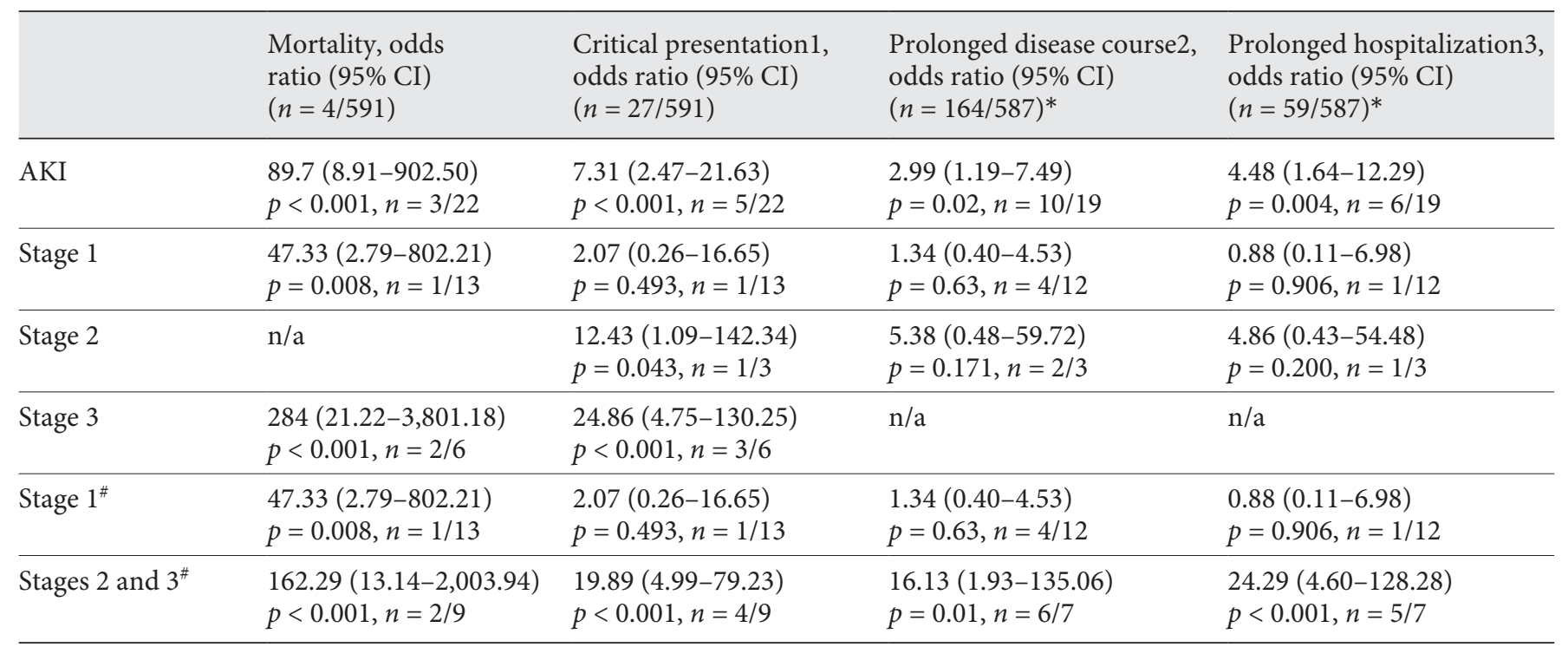

AKI, acute kidney injury. ${ }^{1}$ Defined as intensive care admission. ${ }^{2}$ Defined as interval between symptom onset and discharge more than or equal to 43 days. ${ }^{3}$ Defined as duration of hospitalization more than or equal to 35 days, both equivalent to the 90 th percentile in the Hong Kong cohort. " Regrouped due to small strata of stage 2 and 3 AKI patients. AKI stage defined according to KDIGO 2012. * Excluded 4 patients deceased at the time of censor.

Table 4. Factors associated with in-hospital AKI in the Hong Kong cohort

\begin{tabular}{ll} 
Independent factors & $\begin{array}{l}\text { Odds ratio (OR)/regression coefficient (RC) } \\
(95 \% \mathrm{CI})\end{array}$ \\
\hline Age & RC: $0.050(0.028-0.072), p<0.001$ \\
Ex-smoker & OR: $5.432(1.449-20.362), p=0.012$ \\
Diabetes history & OR: $9.017(3.404-23.887), p<0.001$ \\
Hypertension history & OR: $8.841(3.680-21.243), p<0.001$ \\
CKD history & OR: $3.980(1.489-10.634), p=0.006$ \\
Tumor history & OR: $4.642(0.973-22.133), p=0.054$ \\
Concurrent use of ACE inhibitor/ARB & OR: $5.721(2.108-15.531), p=0.001$ \\
Baseline systolic blood pressure (mm Hg) & RC: $0.287(0.007-0.050), p=0.010$ \\
Baseline C-reactive protein (mg/L) & RC: $0.009(0.004-0.013), p<0.001$ \\
Baseline LDH (U/L) & RC: $0.008(0.004-0.011), p<0.001$ \\
Baseline GFR (mL/min/1.73 $\left.\mathrm{m}^{2}\right)$ & RC: $-0.033(-0.051$ to -0.014$), p=0.001$ \\
Baseline serum albumin (g/L) & RC: $-0.145(-0.222$ to -0.069$), p<0.001$ \\
Baseline creatine kinase (U/L) & RC: $0.005(0.002-0.007), p<0.001$ \\
Bilateral lung infiltration & OR: $6.189(2.190-17.493), p=0.001$ \\
C-reactive protein surpassed 20 mg/L during hospitalization & OR: $3.492(1.477-8.255), p=0.004$ \\
LDH surpassed 280 U/L during hospitalization & OR: $5.182(2.141-12.544), p<0.001$
\end{tabular}

AKI, acute kidney injury; LDH, lactate dehydrogenase; GFR, glomerular filtration rate. Assessed by univariable logistic regression model with in-hospital AKI as dependent variable. 


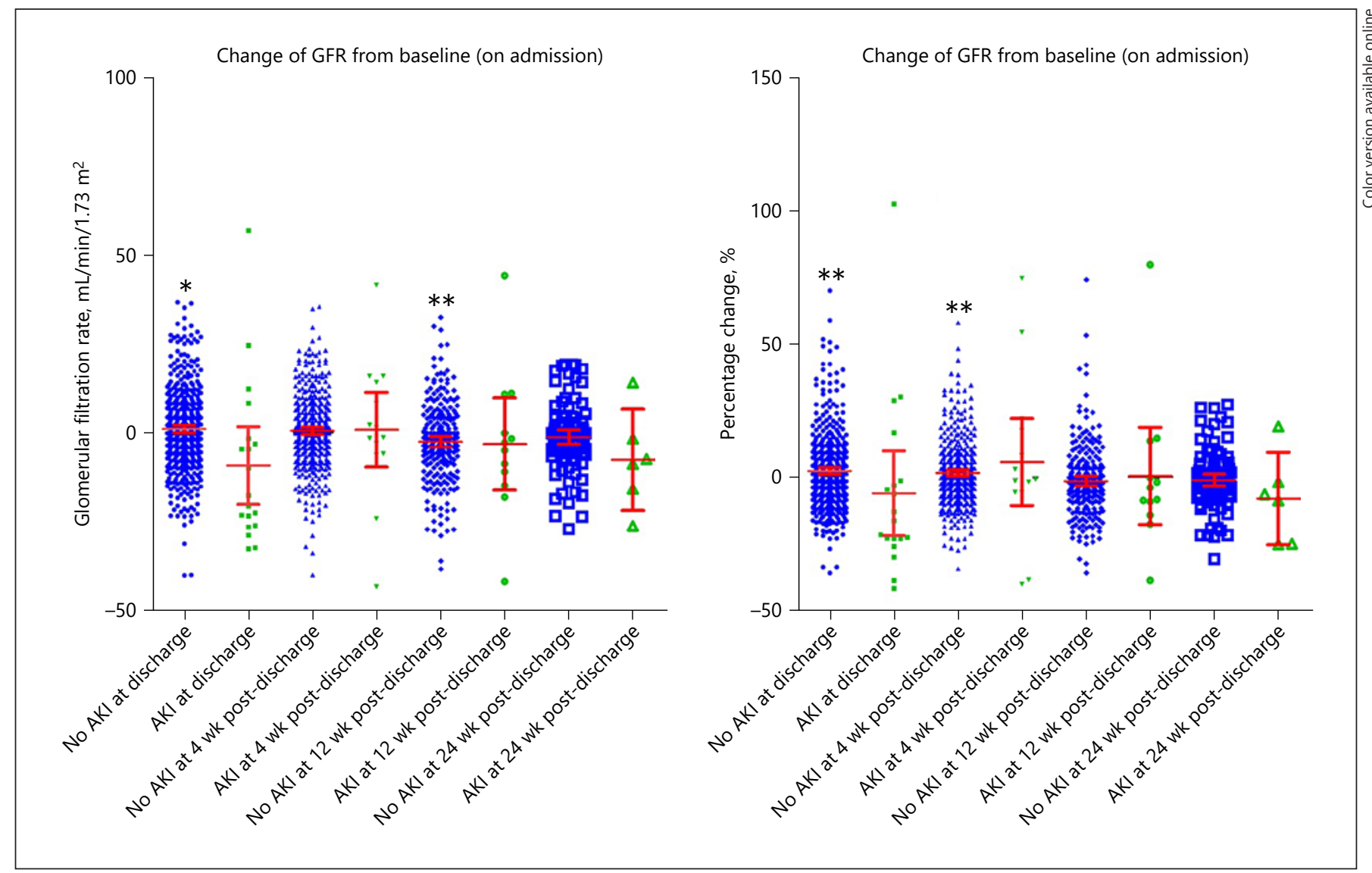

Fig. 2. Mean percentage change of estimated GFR from baseline $\left({ }^{*} p<0.05,{ }^{* *} p<0.01\right)$. Estimated GFR change from baseline. $95 \%$ confidence intervals are shown. GFR was estimated by CKD-EPI equation. Patients who developed AKI during hospitalization had a trend of greater reduction in GFR but not to a statistically significant level. GFR, glomerular filtration rate; AKI, acute kidney injury.

Table 5. Renal manifestation difference between symptomatic and asymptomatic patients

\begin{tabular}{lccc}
\hline & Asymptomatic & Symptomatic & $\begin{array}{l}p \text { value } \\
\text { (asymptomatic vs. } \\
\text { symptomatic) }\end{array}$ \\
\hline Mortality, $n / N(\%)$ & & & 0.352 \\
Intensive care admission, $n / N(\%)$ & $1 / 62(1.6)$ & $3 / 519(0.6)$ & 0.066 \\
AKI, $n / N(\%)$ & $0 / 62(0)$ & $27 / 519(5.2)$ & 0.646 \\
Renal replacement therapy, $n / N(\%)$ & $3 / 62(4.8)$ & $19 / 519(3.7)$ & 0.488 \\
Length of hospitalization, median $[\mathrm{IQR}]$, days & $0 / 62(0)$ & $4 / 519(0.8)$ & $<0.001$ \\
\hline
\end{tabular}

Incidence of AKI was comparable between asymptomatic and symptomatic patients. AKI, acute kidney injury.

lower rate of mortality ( 0.4 vs. $3.9 \%, p=0.004)$, intensive care admission ( 3.9 vs. $11.5 \%, p=0.012$ ), AKI (2.4 vs. $17.3 \%, p<0.001)$, and median length of hospitalization (18 vs. 22.5 days, $p=0.001$ ) than in the 1st wave (Table 6).

\section{Discussion}

Incident in-hospital AKI was associated with increased odds of prolonged disease course, prolonged hospitaliza- 
Table 6. Renal manifestations difference between the 1 st and 2 nd waves

\begin{tabular}{lccc}
\hline & 1st wave & 2nd wave & $\begin{array}{c}p \text { value } \\
\text { (1st vs. 2nd wave) }\end{array}$ \\
\hline Mortality, $n / N(\%)$ & $2 / 52(3.9)$ & $2 / 539(0.4)$ & 0.004 \\
Intensive care admission, $n / N(\%)$ & $6 / 52(11.5)$ & $21 / 539(3.9)$ & 0.012 \\
Acute kidney injury, $n / N(\%)$ & $9 / 52(17.3)$ & $13 / 539(2.4)$ & $<0.001$ \\
Renal replacement therapy, $n / N(\%)$ & $1 / 52(1.9)$ & $3 / 539(0.6)$ & 0.251 \\
Days from symptom onset to admission, median [IQR] & $5.5[3-9]$ & $5[2-9]$ & 0.594 \\
Days from symptom onset to discharge, median [IQR] & $29[23-35]$ & $25[19-33]$ & 0.024 \\
Length of intensive care, median [IQR], days & $9[4-16]$ & $13[9-22]$ & 0.255 \\
Length of hospitalization, median [IQR], days & $22.5[17-31]$ & $18[11-26]$ & 0.001 \\
\hline
\end{tabular}

The disease course and length of hospitalization were shorter during the 2nd wave.

tion, intensive care admission, and mortality among individual patients. The incidence of AKI was comparable between asymptomatic and symptomatic patients. COVID-19 patients' GFR showed a trend of persistent reduction after 24 weeks of discharge.

\section{Unique Characteristics of the Hong Kong Cohort}

The incidence of in-hospital AKI and mortality in the Hong Kong cohort were among the lowest from reported studies from Wuhan and the USA $[17,18]$. However, there were $18.9 \%$ of the AKI patients required RRT, which is comparable to the Wuhan cohort (15\%) reported earlier [17]. From the demographics, our 2nd wave cohort was younger, with less comorbidities, had a shorter time from symptom onset to admission, less symptomatic, and with shorter hospitalization and disease course. Hong Kong had a relatively mild cohort partly due to the effective border and community surveillance that led to early detection and hospital isolation of RT-PCR confirmed cases. These factors contributed to the observation of overall lower severity of patients in our cohort and provided a unique source of information to assess the health services need under strong surveillance when compared to other cohorts globally, which is of high demand lately. Noteworthy, the Hong Kong cohort also had a longer hospitalization period, partly due to the reduced latent period between symptom onset to admission and the repeated negative RT-PCR results before discharge.

\section{Clinical Implication - Prolonged Hospitalization and Disease Course}

Since the outbreak of COVID-19, the overloading of intensive care service, hospital beds, ventilators, and dialysis machines has been widely reported worldwide [19, 20], which was postulated to cause delay in treatment ac- cess, prolong viral shedding, and increase mortality [21, $22]$. The data from the Hong Kong cohort showed that AKI and RRT were associated with 3.5 times increased odds of prolonged hospitalization (over 35 days) and longer intensive care stay (AKI: 26 days vs. non-AKI: 10.5 days), as AKI involves pathophysiology of multiple organs, which leads to more complicated management $[10$, 23]. The prevention of AKI would be important to reduce the overall health service burden, as the incidence of AKI could reach $30-50 \%$ in some settings.

The presentation of AKI in COVID-19 and SARS was different in Hong Kong. The incidence of AKI was lower and earlier in COVID-19 (3.7\%) than in SARS $(6.4 \%)[24,25]$. For COVID-19, the median time to develop AKI after admission was 2 [1-7] days, and the median time from symptom onset and admission to reaching peak SCr were 15 [10-18] and 5 [5-12] days, whereas the median time from the onset of viral infection to AKI was 20 [5-48] days in SARS [24]. In our cohort, SCr reached the peak (median: 15 days) following LDH (median: 9 days) among patients who developed AKI. The increase in serum LDH indicates organ injury including AKI. The chronological sequence suggested that the increase in LDH could partially come from AKI, and $\mathrm{LDH}$ has been proposed as a predictor of AKI previously [26]. The median day to reach peak $\mathrm{LDH}$ was comparable between the AKI and non-AKI groups, and the median days to reach peak C-reactive protein and $\mathrm{SCr}$ were longer in the AKI group, indicating that the progression of AKI could be associated with sustained systemic inflammation [27].

We also identified a series of risk factors associated with the onset of AKI from our cohort (Table 4). Of these factors, lower baseline serum albumin was observed among COVID-19 patients who subsequently developed 
AKI, which was a common risk factor shared with SARS [24]. Strategies to prevent or correct hypoalbuminemia including dietary intervention or albumin administration could be potential trial interventions. Evidence on more amenable factors that associated with faster and better AKI recovery is limited, requiring urgent clarification. A recent review showed that the mortality of COVID-19 AKI patients reached $76.5 \%$ and was comparable to that of SARS (86.6\%) and MERS (68.5\%) [28]. The 1-year mortality hazard ratio among AKI survivors in previous studies varied from 1.63 to 2.48 , positively correlated to the preceding CKD stage [12].

The use of ACE inhibitors/ARB was found associated with increased odds of developing AKI in our cohort, which reconciled with other studies [29]. However, the magnitude of association between diabetes, hypertension, and AKI was greater, and the use of ACE inhibitors/ ARB was more likely in the causal pathway instead of being the root cause of AKI. Previous retrospective studies showed that discontinuing ACE inhibitors/ARB was associated with increased risk of AKI among COVID-19 patients [30]. Preliminary data of the BRACE CORONA trial also showed similar results [31]. Nevertheless, evidence on the mortality and AKI incidence associated with different add-on medications for hypertension management in COVID-19 is lacking.

\section{Urgency of Strategies to Speed Up AKI Recovery before} Strong Surveillance

Early detection could lead to earlier treatment and therefore lower the severity of COVID-19 patients [32]. Nevertheless, early detection would lead to an exponential increase in positive mild cases and increased hospital service load. Therefore, strategies to shorten hospital stay, mitigate transmission, and control the overall incidence of COVID-19 are crucial, should mass surveillance be adopted. To this end, efficient AKI management is critical. Our cohort showed that although increased surveillance did not increase the average hospitalization period, AKI was associated with substantial increased risk of prolonged hospitalization among individuals. Evidence on strategies to fasten the recovery of AKI is lacking, yet of paramount importance. Targeted protection and screening on vulnerable patient groups, for example, the elderly, diabetic, hypertensive, and patients with chronic diseases, are needed, as these factors are also significantly associated with AKI. To shorten hospital stay, previous studies demonstrated the early use of interferon may shorten the disease course [32].

\section{Current Prevention and Management of AKI in COVID-19}

Management of AKI in COVID-19 should be individualized depending on the overall hemodynamic and respiratory status. In the absence of high-quality evidence, it is reasonable to take into consideration a recently proposed lung-kidney interaction model that advocates mitigating volutrauma and barotrauma through adopting lung-protective ventilation approaches [33]. This is believed to lower the risk of incident or worsening AKI by limiting ventilation-induced hemodynamic disturbance and a putative cytokine burden on the kidney.

For the prevention of AKI, an important aspect is early recognition of abnormal fluid balance [34]. In the Hong Kong cohort, the rate of in-hospital AKI was comparable between symptomatic and asymptomatic patients, suggesting AKI may not be timely recognized if asymptomatic patients were not admitted. Prompt volume correction may avert AKI, as volume depletion on admission might be common in patients with COVID-19, given that they typically present with fever and prehospital fluid resuscitation is rarely performed [35]. Stringent infection control and early treatment for high-risk individuals are other important measures, as patients had the highest viral load near presentation [36], and early triple antiviral therapy shortens the duration of viral shedding and hospital stay [32], potentially reducing the chance of renal endothelial cell damage by viremia, progression to overwhelming sepsis and a multi-organ dysfunction syndrome [35].

\section{Long-Term Renal Involvement after COVID-19}

$\mathrm{AKI}$ and the need of RRT also have significant implication to the health service provision post-outbreak. AKI is associated with 1.7 and 3.81 times increased hazard for developing CKD and end-stage kidney disease [37], and $79 \%$ of RRT patients did not recover 6 months after discharge, as reported in previous studies [38]. In our cohort, patients who developed in-hospital AKI had a trend of persistent GFR reduction after 24 weeks of discharge when compared to patients who did not have AKI, which is consistent with the Wuhan cohort (32\% non-recoverable at discharge) $[17,39]$. As 1-year interval was previously shown to divide the hazard of mortality [12], adequate provision of renal follow-up service within the first 12 months would be critical to reduce the intermediateterm increase in mortality. The residual renal impairment at population level, especially incident end-stage kidney disease, is expected to substantially increase the demand for long-term renal care including dialysis, for which the access could be limited and costly [40]. 


\section{Limitations}

In the retrospective cohort, we only analyzed key manifestations including AKI and RRT, as the records on these 2 outcomes were more complete among patients. We did not analyze the incidence and effect of proteinuria and hematuria and other biomarkers (e.g., glycated hemoglobin, lipids, and troponins), as they were not routinely tested for all COVID-19 patients in our setting and could lead to substantial selection bias. Also, information on the comparison between AKI developed based on urine output changes versus $\mathrm{Cr}$ and both is limited and underpowered. Besides, the quantitative viral load was not routinely available in the electronic medical record. The association between viral load on admission and key clinical outcomes requires further research. Since our cohort captured a unique pool of younger patients with less comorbidity, majority did not have prehospital SCr records. Prehospital AKI could not be assessed, and the incidence of AKI may be underestimated. The number of patients with in-hospital AKI was small, and therefore, multivariable regression analysis on risk factors was conducted as a supplementary analysis, and the confidence intervals of the odds ratios are large. Last, only a portion of patients attended follow-up and the analysis is underpowered. However, the incompleteness of follow-up was due to standard protocol of follow-up cessation and the relocation of overseas patients, and therefore, selection bias is minimal.

\section{Conclusion}

The overall rate of AKI among COVID-19 patients in Hong Kong is low, which could be attributable to a vigilant screening program and early hospitalization. Among patients who developed in-hospital AKI, the duration of hospitalization is prolonged and kidney function impairment can persist for up to 6 months post-discharge. Mass surveillance for COVID-19 is warranted in identifying asymptomatic subjects for earlier AKI management.

\section{Acknowledgements}

We thank the philanthropic donations from Mr. Winston Leung, Mr. Kwok Keung Chan, Dr. Ying Yau Cheung, and Ms. Siu Suet Lau and an Endowment Fund established at the University of Hong Kong for the Yu Professorship in Nephrology awarded to S.C.-W.T. We also thank Ms. Pearl Yun for the clerical support on the data extraction.

\section{Statement of Ethics}

This study was approved by the Institutional Review Boards of the 5 corresponding hospital clusters of the Hospital Authority Hong Kong via Central Coordination Office (Ref.: CCO-20200011). This study is reported according to STROBE.

\section{Conflict of Interest Statement}

None declared. Part of the results in the article has been accepted as poster presentation at the American Society of Nephrology Kidney Week 2020.

\section{Funding Sources}

This project is made possible in part through the Health and Medical Research Fund (Ref.: 14151731). The funding organization had no role in the design and conduct of the study; collection, management, analysis, and interpretation of the data; preparation, review, or approval of the manuscript; and decision to submit the manuscript for publication.

\section{Author Contributions}

S.C.-W.T. and K.W.C. conceived the study. I.F.-N.H., O.T.Y.T., T.C.W., E.Y.-K.T., K.C.L., G.C.W.-C., S.S.-H.W., and J.W.M.C. recruited the patients and provided clinical service. K.Y.Y. and K.W.C. retrieved the cohort data. K.W.C. performed the statistical analyses. K.W.C. and S.C.-W.T. drafted the manuscript. All authors proofread the manuscript.

\section{References}

Renal Involvement in COVID-19 in Hong Kong
1 Pei G, Zhang Z, Peng J, Liu L, Zhang C, Yu C, et al. Renal involvement and early prognosis in patients with COVID-19 pneumonia. J Am Soc Nephrol. 2020;31(6):1157-65.

2 Wu Z, McGoogan JM. Characteristics of and important lessons from the coronavirus disease 2019 (COVID-19) outbreak in China: summary of a report of 72,314 cases from the Chinese center for disease control and prevention. JAMA. 2020;323(13):1239-42.
3 Yang X, Yu Y, Xu J, Shu H, Xia J, Liu H, et al Clinical course and outcomes of critically ill patients with SARS-CoV-2 pneumonia in Wuhan, China: a single-centered, retrospective, observational study. Lancet Respir Med. 2020;8(5):475-81. 
4 Geographic Differences in COVID-19 Cases, Deaths, and Incidence - United States, Centre for Disease Control and Prevention; 2020. https://www.cdc.gov/mmwr/volumes/69/wr/ $\mathrm{mm} 6915 \mathrm{e} 4 . \mathrm{htm}$ ?s_cid $=\mathrm{mm} 6915 \mathrm{e} 4$ $\mathrm{w} \#$ References.

5 Karagiannidis C, Mostert C, Hentschker C, Voshaar T, Malzahn J, Schillinger G, et al. Case characteristics, resource use, and outcomes of 10,021 patients with COVID-19 admitted to 920 German hospitals: an observational study. Lancet Respir Med. 2020;8(9): 853-62.

6 Adam DC, Wu P, Wong JY, Lau EHY, Tsang TK, Cauchemez S, et al. Clustering and superspreading potential of SARS-CoV-2 infections in Hong Kong. Nat Med. 2020;26(11): 1714-9.

7 Peto J, Alwan NA, Godfrey KM, Burgess RA, Hunter DJ, Riboli E, et al. Universal weekly testing as the UK COVID-19 lockdown exit strategy. Lancet. 2020;395(10234):1420-1.

8 Braithwaite I, Callender T, Bullock M, Aldridge RW. Automated and partly automated contact tracing: a systematic review to inform the control of COVID-19. Lancet Digit Health. 2020;2(11):E607-21.

9 Peak CM, Kahn R, Grad YH, Childs LM, Li R, Lipsitch $\mathrm{M}$, et al. Individual quarantine versus active monitoring of contacts for the mitigation of COVID-19: a modelling study. Lancet Infect Dis. 2020;20(9):1025-33.

10 Ronco C, Bellomo R, Kellum JA. Acute kidney injury. Lancet. 2019;394(10212):1949-64.

11 Sawhney S, Fraser SD. Epidemiology of AKI: utilizing large databases to determine the burden of AKI. Adv Chronic Kidney Dis. 2017; 24(4):194-204.

12 Sawhney S, Marks A, Fluck N, Levin A, Prescott G, Black C. Intermediate and longterm outcomes of survivors of acute kidney injury episodes: a Large Population-Based Cohort Study. Am J Kidney Dis. 2017;69(1): $18-28$.

13 Challiner R, Ritchie JP, Fullwood C, Loughnan $\mathrm{P}$, Hutchison AJ. Incidence and consequence of acute kidney injury in unselected emergency admissions to a large acute UK hospital trust. BMC Nephrol. 2014;15:84.

14 Kidney Disease: Improving Global Outcomes (KDIGO) Acute Kidney Injury Work Group.. KDIGO Clinical Practice Guideline for Acute Kidney Injury. Kidney Int Suppl. 2012;2:1138.

15 Levey AS, Stevens LA, Schmid CH, Zhang YL, Castro AF 3rd, Feldman HI, et al. A new equation to estimate glomerular filtration rate. Ann Intern Med. 2009;150(9):604-12.

16 Latest Situation of Coronavirus Disease (COVID-19) in Hong Kong [Internet]. 2020 [cited 2020 Oct 20]. Available from: https://chpdashboard.geodata.gov.hk/covid-19/en.html.
17 Siew ED, Birkelo BC. COVID-19-associated acute kidney injury: an evolving picture. Clin J Am Soc Nephrol. 2020;15(10):1383-5.

18 Fisher M, Neugarten J, Bellin E, Yunes M, Stahl L, Johns TS, et al. AKI in hospitalized patients with and without COVID-19: a Comparison Study. J Am Soc Nephrol. 2020; 31(9):2145-57.

19 Murray CJ. Forecasting COVID-19 impact on hospital bed-days, ICU-days, ventilator-days and deaths by US state in the next 4 months. medRxiv. 2020.

20 Remuzzi A, Remuzzi G. COVID-19 and Italy: what next? Lancet. 2020;395(10231):1225-8.

21 Zhou F, Yu T, Du R, Fan G, Liu Y, Liu Z, et al. Clinical course and risk factors for mortality of adult inpatients with COVID-19 in Wuhan, China: a retrospective cohort study. Lancet. 2020;395(10229):1054-62.

22 Wang Y, Guo Q, Yan Z, Zhou D, Zhang W, Zhou $S$, et al. Factors associated with prolonged viral shedding in patients with avian influenza A (H7N9) virus infection. J Infect Dis. 2018;217(11):1708-17.

23 Husain-Syed F, McCullough PA, Birk HW, Renker M, Brocca A, Seeger W, et al. Cardiopulmonary-renal interactions: a multidisciplinary approach. J Am Coll Cardiol. 2015; 65(22):2433-48.

24 Chu KH, Tsang WK, Tang CS, Lam MF, Lai FM, To KF, et al. Acute renal impairment in coronavirus-associated severe acute respiratory syndrome. Kidney Int. 2005;67(2):698705.

25 Choi KW, Chau TN, Tsang O, Tso E, Chiu $\mathrm{MC}$, Tong WL, et al. Outcomes and prognostic factors in 267 patients with severe acute respiratory syndrome in Hong Kong. Ann Intern Med. 2003;139(9):715-23.

26 Coca SG, Yalavarthy R, Concato J, Parikh CR Biomarkers for the diagnosis and risk stratification of acute kidney injury: a systematic review. Kidney Int. 2008;73(9):1008-16.

27 Yan L, Zhang H-T, Goncalves J, Xiao Y, Wang M, Guo Y, et al. An interpretable mortality prediction model for COVID-19 patients. Nat Mach Intell. 2020;2(5):283-8.

28 Chen YT, Shao SC, Lai EC, Hung MJ, Chen YC. Mortality rate of acute kidney injury in SARS, MERS, and COVID-19 infection: a systematic review and meta-analysis. Crit Care. 2020;24(1):439.

29 Oussalah A, Gleye S, Clerc Urmes I, Laugel E, Callet J, Barbé F, et al. Long-term ACE inhibitor/ARB use is associated with severe renal dysfunction and acute kidney injury in patients with severe COVID-19: results from a referral center cohort in the Northeast of France. Clin Infect Dis. 2020;71(9):2447-56.
30 Lam KW, Chow KW, Vo J, Hou W, Li H, Richman PS, et al. Continued in-hospital angiotensin-converting enzyme inhibitor and angiotensin II receptor blocker use in hypertensive COVID-19 patients is associated with positive clinical outcome. J Infect Dis. 2020; 222(8):1256-64.

31 Lopes RD, Macedo AVS, de Barros E Silva PGM, Moll-Bernardes RJ, Feldman A, D'Andréa Saba Arruda G, et al. Continuing versus suspending angiotensin-converting enzyme inhibitors and angiotensin receptor blockers: impact on adverse outcomes in hospitalized patients with severe acute respiratory syndrome coronavirus 2 (SARS-CoV-2): the BRACE CORONA trial. Am Heart J. 2020; 226:49-59.

32 Hung IF-N, Lung K-C, Tso EY-K, Liu R, Chung TW-H, Chu M-Y, et al. Triple combination of interferon beta-1b, lopinavir-ritonavir, and ribavirin in the treatment of patients admitted to hospital with COVID-19: an open-label, randomised, phase 2 trial. The Lancet. 2020;395(10238):1695-704.

33 Joannidis M, Forni LG, Klein SJ, Honore PM, Kashani K, Ostermann M, et al. Lung-kidney interactions in critically ill patients: consensus report of the acute disease quality initiative (ADQI) 21 Workgroup. Intensive Care Med. 2020;46(4):654-72.

34 Levey AS, James MT. Acute kidney injury. Ann Intern Med. 2017;167(9):Itc66-tc80.

35 Ronco C, Reis T, Husain-Syed F. Management of acute kidney injury in patients with COVID-19. Lancet Respir Med. 2020;8(7): $738-42$.

36 To KK, Tsang OT, Leung WS, Tam AR, Wu TC, Lung DC, et al. Temporal profiles of viral load in posterior oropharyngeal saliva samples and serum antibody responses during infection by SARS-CoV-2: an observational cohort study. Lancet Infect Dis. 2020;20(5):56574.

37 See EJ, Jayasinghe K, Glassford N, Bailey M, Johnson DW, Polkinghorne KR, et al. Longterm risk of adverse outcomes after acute kidney injury: a systematic review and metaanalysis of cohort studies using consensus definitions of exposure. Kidney Int. 2019; 95(1):160-72.

38 Hickson LJ, Chaudhary S, Williams AW, Dillon JJ, Norby SM, Gregoire JR, et al. Predictors of outpatient kidney function recovery among patients who initiate hemodialysis in the hospital. Am J Kidney Dis. 2015;65(4): 592-602.

39 Cheng Y, Luo R, Wang X, Wang K, Zhang N, Zhang $\mathrm{M}$, et al. The incidence, risk factors, and prognosis of acute kidney injury in adult patients with coronavirus disease 2019. Clin J Am Soc Nephrol. 2020;15(10):1394-402.

40 Teerawattananon Y, Dabak SV, Khoe LC, Bayani DBS, Isaranuwatchai W. To include or not include: renal dialysis policy in the era of universal health coverage. BMJ. 2020;368: m82. 